\title{
Contact properties of codimension 2 submanifolds with flat normal bundle
}

\section{J. J. Nuño-Ballesteros and M. C. Romero-Fuster}

\begin{abstract}
Given an immersed submanifold $M^{n} \subset \mathbb{R}^{n+2}$, we characterize the vanishing of the normal curvature $R_{D}$ at a point $p \in M$ in terms of the behaviour of the asymptotic directions and the curvature locus at $p$. We relate the affine properties of codimension 2 submanifolds with flat normal bundle with the conformal properties of hypersurfaces in Euclidean space. We also characterize the semiumbilical, hypespherical and conformally flat submanifolds of codimension 2 in terms of their curvature loci.
\end{abstract}

\section{Introduction}

The analysis of the singularities of height and distance-squared functions has proven to be useful in the study of the extrinsic geometry of submanifolds $([5,7,8,12,13])$. In fact, some well known geometrical concepts, such as principal and asymptotic configurations, or focal subsets, arise in a natural way in this context. More precisely, we can view the principal directions on a hypersurface $M$ of Euclidean space $\mathbb{R}^{n}$ as those lying in the kernel of the Hessian quadratic form of some distance-squared function on $M$. On the other hand, the asymptotic directions on codimension two submanifolds of $\mathbb{R}^{n}$ can be characterized as kernel directions of the hessian of height functions (see [8]). The properties related to the singularities of distance-squared functions, and thus to the contacts with (or approximations by) hyperspheres is called here the round extrinsic geometry, whereas those arising from the singularities of height functions, related in turn with the contacts with (or approximation by) hyperplanes, is the flat extrinsic geometry. An interesting fact is that stereographic projection transforms

2000 Mathematics Subject Classification: 53A05, 58C25.

Keywords: Asymptotic directions, $\nu$-principal curvature foliation, umbilicity, sphericity, normal curvature. 
contacts of hypersurfaces of $S^{n}$ with hyperplanes in $\mathbb{R}^{n+1}$ into contacts of hypersurfaces in $\mathbb{R}^{n}$ with hyperspheres [14], and thus provides a "bridge" between the flat geometry of hypersurfaces of $S^{n}$ and the round geometry of the hypersurfaces of $\mathbb{R}^{n}$.

The hypersurfaces of $S^{n}$ considered as a particular case of codimension two submanifolds of $\mathbb{R}^{n+1}$ satisfy the especial property of having a unique principal configuration. In this sense they form part of a more general class of submanifolds: Codimension two submanifolds with flat normal bundle, or equivalently, with vanishing normal curvature. We concentrate here in this more general class of submanifolds and introduce an alternative to the stereographic projection showing that the round extrinsic geometry of hypersurfaces in $\mathbb{R}^{n}$ is equivalent to the flat extrinsic geometry of the submanifolds of codimension two with vanishing normal curvature in $\mathbb{R}^{n+1}$.

Our aim in this paper consists in exploring this relation in order to obtain new results on submanifolds of codimension 2 with vanishing normal curvature in Euclidean space from a global viewpoint.

The case of surfaces in 4-space has been extensively studied from different viewpoints (see for instance $[6,9,7,15,17]$ ). The main tool used in all these works is the analysis of several concepts arising from the second fundamental form: curvature ellipses, shape operators associated to normal fields and their associated principal configurations, asymptotic direction fields, normal curvature, etc. An interesting fact is the equivalence among the following properties at a given point of the surface:

a) Vanishing normal curvature.

b) Degeneracy of the curvature ellipse.

c) Critical point of some principal configuration.

d) Singularity of corank 2 for some squared distance function.

e) Existence of two orthogonal asymptotic directions.

In the present paper we start by investigating the generalization of these equivalences to submanifolds of codimension 2 in Euclidean space. We introduce here the concept of curvature locus at each point as a natural generalization of the curvature ellipse at a point of a surface immersed in $\mathbb{R}^{n}$. We see that this is a convex subset in the normal space of the submanifold at the given point. This subset becomes a convex polygon at points at which the normal curvature of the submanifold vanishes (Corollary 4.4). We analyze with detail the codimension 2 submanifolds with vanishing normal curvature. A consequence of well known properties of such submanifolds is the fact that all their shape operators share an orthogonal basis of principal directions. We explore here this fact in order to show that they admit an orthogonal basis of asymptotic directions at each point (Theorem 3.3). 
The asymptotic directions were introduced in [7] for surfaces immersed in $\mathbb{R}^{4}$ and in [8] for the general case of submanifolds of codimension 2 in Euclidean space. Their definition was given in terms of the contacts of the submanifolds with the hyperplanes of the ambient space, and thus characterized in terms of the singularities of the height functions on the submanifolds. It was shown in [8] that any $n$-manifold immersed in $\mathbb{R}^{n+2}$ admits at most $n$ asymptotic directions at each one of its points. Moreover, a sufficient condition for the existence of some asymptotic direction at a point is the local convexity. We show in Section 5 that although the strict local convexity is a sufficient condition for the existence of the maximal number of asymptotic directions at a point, it does not imply the vanishing of the normal curvature at the given point. Nor the vanishing of the normal curvature implies strict local convexity.

Since the inverse of the stereographic projection from $\mathbb{R}^{n}$ to $S^{n}$ transforms the principal curvature lines of a hypersurface in $\mathbb{R}^{n}$ into the asymptotic lines of its image into $S^{n}$ (considered as a codimension 2 submanifold of $\mathbb{R}^{n+1}$ (see [8]), we have that codimension 2 submanifolds contained in hyperspheres of $\mathbb{R}^{n+1}$ have the maximal number $(=n-1)$ of mutually orthogonal asymptotic directions at every point. Our result implies that this fact extends to the more general class of submanifolds of codimension 2 with vanishing normal curvature. In fact, we show that the existence of an orthonormal basis of asymptotic directions for the tangent space at a point is equivalent to the vanishing of the normal curvature of the submanifold at the given point (Theorem 3.3). An important particular case of codimension 2 submanifolds with vanishing normal curvature consists of the semiumbilical submanifolds, that is, submanifolds all whose points are $\nu$-umbilics, for some normal field $\nu$. We show that for these, the curvature locus becomes a segment whose direction is orthogonal to $\nu$. We observe that though this property characterizes the vanishing of the normal curvature for surfaces immersed in 4-space, this is not the case in higher dimensions.

In Section 6 we use the fact that codimension 2 submanifolds with vanishing normal curvature have some parallel normal field in order to show that the study of the contacts of such submanifolds with hyperplanes (leading to affinely invariant properties) is equivalent to the study of the contacts of hypersurfaces with hyperspheres. In other words, there is an equivalence between affinely invariant properties of n-manifolds with vanishing normal curvature in $\mathbb{R}^{n+2}$ and the conformally invariant properties of hypersurfaces in $\mathbb{R}^{n+1}$.

A special case of semiumbilical submanifolds is given by those contained in a hypersphere ([1]). We analyze in Section 7 some necessary and sufficient conditions for the hypersphericity of a semiumbilical submanifold of 
codimension 2 in terms of the properties of the curvature locus and the asymptotic directions. We also analyze the conformal flatness of semiumbilical submanifolds in this context.

\section{Vanishing normal curvature and principal configura- tions}

Let $M$ be a smooth immersed $n$-dimensional manifold in $\mathbb{R}^{n+k}$. We consider in $M$ the Riemannian metric induced by the Euclidean metric of $\mathbb{R}^{n+k}$. Given a point $p \in M$, we have a decomposition

$$
\mathbb{R}^{n+k}=T_{p} M \oplus N_{p} M,
$$

where $N_{p} M=T_{p} M^{\perp}$ and the corresponding orthogonal projections $\top$ : $\mathbb{R}^{n+k} \rightarrow T_{p} M$ and $\perp: \mathbb{R}^{n+k} \rightarrow N_{p} M$.

We denote by $\nabla^{\prime}$ the covariant derivative in $\mathbb{R}^{n+k}$. For vector fields $X, Y$ tangent along $M$ in a neighbourhood of $p$, we have the Gauss formula,

$$
\nabla_{X}^{\prime} Y=\nabla_{X} Y+s(X, Y)
$$

where

1. $\nabla$ is the induced covariant derivative in $M$ given by $\nabla_{X} Y=\top\left(\nabla_{X}^{\prime} Y\right)$.

2. $s$ is the second fundamental form, that is, $s: T_{p} M \times T_{p} M \rightarrow N_{p} M$ is the bilinear symmetric map defined by $s(X, Y)=\perp\left(\nabla_{X}^{\prime} Y\right)$.

We denote the curvature tensor of $\nabla$ by $R: T_{p} M \times T_{p} M \times T_{p} M \rightarrow T_{p} M$, which is defined as

$$
R(X, Y) Z=\nabla_{X}\left(\nabla_{Y} Z\right)-\nabla_{Y}\left(\nabla_{X} Z\right)-\nabla_{[X, Y]} Z .
$$

Since the curvature of $\nabla^{\prime}$ in $\mathbb{R}^{n+k}$ is identically zero, we can compute $R$ by using the Gauss equation:

$$
\langle R(X, Y) Z, W\rangle=\langle s(X, W), s(Y, Z)\rangle-\langle s(X, Z), s(Y, W)\rangle .
$$

Analogously, if now $\nu$ is a normal vector field along $M$ in a neighbourhood of $p$, we have the Weingarten equation:

$$
\nabla_{X}^{\prime} \nu=-A_{\nu}(X)+D_{X} \nu
$$

where

1. $D$ is the covariant derivative of the normal bundle of $M$, given by $D_{X} \nu=\perp\left(\nabla_{X}^{\prime} \nu\right)$. 
2. For each normal vector $\nu \in T_{p} M^{\perp}, A_{\nu}$ is the shape operator, that is, it is the self-adjoint linear map $A_{\nu}: T_{p} M \rightarrow T_{p} M$ defined by $A_{\nu}(X)=-\top\left(\nabla_{X}^{\prime} \nu\right)$. It is related to the second fundamental form by

$$
\left\langle A_{\nu}(X), Y\right\rangle=\langle s(X, Y), \nu\rangle
$$

for any $X, Y \in T_{p} M$.

Finally, we can also consider the curvature tensor of the normal bundle connection, $R_{D}: T_{p} M \times T_{p} M \times N_{p} M \rightarrow N_{p} M$, given by

$$
R_{D}(X, Y) \nu=D_{X}\left(D_{Y} \nu\right)-D_{Y}\left(D_{X} \nu\right)-D_{[X, Y]} \nu
$$

Since $\mathbb{R}^{n+k}$ has vanishing curvature, the Ricci equation gives

$$
\left\langle\xi, R_{D}(X, Y) \nu\right\rangle=\left\langle X,\left(A_{\xi} \circ A_{\nu}-A_{\nu} \circ A_{\xi}\right) Y\right\rangle
$$

for any $X, Y \in T_{p} M$ and $\xi, \nu \in N_{p} M$.

Definition 2.1. Let $\nu \in N_{p} M$ be a normal vector. We consider the selfadjoint linear map $A_{\nu}: T_{p} M \rightarrow T_{p} M$. The eigenvalues $\mu_{1}, \ldots, \mu_{n}$ of $A_{\nu}$ are called $\nu$-principal curvatures of $M$ at $p$.

We say that $X \in T_{p} M$ is an $\nu$-principal direction if it is an eigenvector of $A_{\nu}$. It is well known that for any $\nu \in N_{p} M$ we can choose an orthonormal frame $\left\{X_{1}, \ldots, X_{n}\right\}$ of $T_{p} M$ made of $\nu$-principal directions, that is, such that

$$
A_{\nu}\left(X_{i}\right)=\mu_{i} X_{i}, \quad i=1, \ldots, n .
$$

A point $p \in M$ is said to be $\nu$-umbilic if there is $\mu \in \mathbb{R}$ such that $A_{\nu}=\mu \mathrm{Id}$, or equivalently, if the $\nu$-principal curvatures are equal. We say that $p$ is umbilic if it is $\nu$-umbilic for any $\nu \in N_{p} M$. We say that $p$ is semiumbilic if it is $\nu$-umbilic for some non-zero $\nu \in N_{p} M$.

Remark 2.2. It is well known that for a point $p$ in a submanifold $M^{n}$ of $\mathbb{R}^{n+k}$, the following conditions are equivalent (see [16]):

1. $R_{D}(p)=0$.

2. $A_{\nu} \circ A_{\xi}=A_{\xi} \circ A_{\nu}$, for any $\nu, \xi \in N_{p} M$.

3. There is an orthonormal frame $\left\{X_{1}, \ldots, X_{n}\right\}$ of $T_{p} M$ made of $\nu$-principal directions, for any $\nu \in N_{p} M$. 


\section{Binormal and asymptotic directions}

In this section, we restrict ourselves to the codimension 2 case. That is, we consider $M^{n} \subset \mathbb{R}^{n+2}$, an immersed submanifold. In this codimension, it is very natural to look at the binormal and asymptotic directions, since we have generically a finite number of such directions. These concepts have been studied by Mochida, Ruas and the second author in [7] for surfaces (i.e., $n=2$ ) and in [8] for general $n$.

Definition 3.1. We say that $\nu \in N_{p} M$ is a binormal vector if $\operatorname{det} A_{\nu}=0$, where $A_{\nu}: T_{p} M \rightarrow T_{p} M$. We say that $X \in T_{p} M$ is an asymptotic direction if there is a non-zero binormal vector $\nu \in N_{p} M$ such that $X \in \operatorname{ker} A_{\nu}$.

We remark that in $[7,8]$ the binormal and asymptotic directions have been introduced in a different way, in terms of singularities of height functions. Given a non zero normal vector $\nu \in N_{p} M$, we denote by $h_{\nu}: M \rightarrow \mathbb{R}$ the height function, defined by $h_{\nu}(x)=\langle x, \nu\rangle$.

Moreover, we also see that binormal and asymptotic directions are also related to the generalized eigenvalue problem of the second fundamental form. Let $A, B$ be two $n \times n$ matrices. We say that $(p, q) \in \mathbb{R}^{2} \backslash\{0\}$ is a generalized eigenvalue of $(A, B)$ if $\operatorname{det}(p A+q B)=0$. Analogously, we say that $x \in \mathbb{R}^{n}$ is an associated generalized eigenvector if $(p A+q B) x=0$.

In general, $\operatorname{det}(p A+q B)$ is a homogeneous polynomial of degree $n$ in the variables $p, q$. Thus, if $\operatorname{det}(p A+q B)$ is not identically zero, we may have up to $n$ generalized eigenvalues. Moreover, if $\operatorname{dim} \operatorname{ker}(p A+q B)=r$, then we have that $(p, q)$ is a root of $\operatorname{det}(p A+q B)$ of multiplicity $r$. In such case we say that the generalized eigenvalue $(p, q)$ has multiplicity $r$.

We take $\left\{\nu_{1}, \nu_{2}\right\}$ a frame of $N_{p} M$ and $\left\{X_{1}, \ldots, X_{n}\right\}$ a frame of $T_{p} M$. Assume that

$$
A_{\nu_{1}}\left(X_{i}\right)=\sum_{j} a_{i j} X_{j}, \quad A_{\nu_{2}}\left(X_{i}\right)=\sum_{j} b_{i j} X_{j}
$$

and let us denote the coefficient matrices by $A=\left(a_{i j}\right)$ and $B=\left(b_{i j}\right)$.

Lemma 3.2. Given a normal vector $\nu=p \nu_{1}+q \nu_{2}$ and a tangent vector $X=x_{1} X_{1}+\cdots+x_{n} X_{n}$, we have the following equivalences:

1. $\nu$ is binormal and $X$ is an asymptotic direction.

2. $h_{\nu}$ has a degenerated (i.e., non Morse) singularity at $p$ and $X$ belongs to the kernel of the Hessian.

3. $(p, q)$ is a generalized eigenvalue of $(A, B)$ and $\left(x_{1}, \ldots, x_{n}\right)$ is a generalized eigenvector. 
Proof. The equivalence between (1) and (2) follows from

$$
D_{p}^{2} h_{\nu}(X, Y)=\left\langle A_{\nu}(X), Y\right\rangle=\langle s(X, Y), \nu\rangle, \quad \forall X, Y \in T_{p} M,
$$

where $D_{p}^{2} h_{\nu}: T_{p} M \times T_{p} M \rightarrow \mathbb{R}$ is the second differential of $h_{\nu}$. The equivalence between (1) and (3) follows directly from the definitions, since $A_{\nu}=p A_{\nu_{1}}+q A_{\nu_{2}}$.

Given a surface $M^{2} \subset \mathbb{R}^{4}$, it was shown in [15] that $p$ is semiumbilic if and only if there are two asymptotic directions which are orthogonal. We see that this can be generalized to higher dimensions, although we have to replace semiumbilicity with the condition that $R_{D}(p)=0$ (we refer to next section for the relationship between these two concepts).

Theorem 3.3. Let $M^{n} \subset \mathbb{R}^{n+2}$ be an immersed submanifold and let $p \in M$. Then $R_{D}(p)=0$ if and only if there is an orthonormal frame $\left\{X_{1}, \ldots, X_{n}\right\}$ of $T_{p} M$ made of asymptotic directions.

Proof. Assume that $R_{D}(p)=0$. By Remark 2.2, there is an orthonormal frame $\left\{X_{1}, \ldots, X_{n}\right\}$ of $T_{p} M$ made of $\nu$-principal directions, for any $\nu \in N_{p} M$. If $\left\{\nu_{1}, \nu_{2}\right\}$ is an orthonormal frame of $N_{p} M$, we have

$$
A_{\nu_{1}}\left(X_{i}\right)=\lambda_{i} X_{i}, \quad A_{\nu_{2}}\left(X_{i}\right)=\mu_{i} X_{i}
$$

for $i=1, \ldots, n$. This gives that

$$
\operatorname{det} A_{p \nu_{1}+q \nu_{2}}=\left(p \lambda_{1}+q \mu_{1}\right) \ldots\left(p \lambda_{n}+q \mu_{n}\right) .
$$

Let $i=1, \ldots, n$ and let $\left(p_{i}, q_{i}\right) \neq(0,0)$ such that $p_{i} \lambda_{i}+q_{i} \mu_{i}=0$. Then, $\nu=p_{i} \nu_{1}+q_{i} \nu_{2}$ is a non-zero binormal vector and

$$
A_{\nu}\left(X_{i}\right)=p_{i} A_{\nu_{1}}\left(X_{i}\right)+q_{i} A_{\nu_{2}}\left(X_{i}\right)=\left(p_{i} \lambda_{i}+q_{i} \mu_{i}\right) X_{i}=0,
$$

so that $X_{i}$ is an asymptotic direction.

Let us see the converse. Consider an orthonormal frame $\left\{X_{1}, \ldots, X_{n}\right\}$ of $T_{p} M$ made of asymptotic directions and let $\left\{\nu_{1}, \nu_{2}\right\}$ be an orthonormal frame of $N_{p} M$. We assume that

$$
A_{\nu_{1}}\left(X_{i}\right)=\sum_{j} a_{i j} X_{j}, \quad A_{\nu_{2}}\left(X_{i}\right)=\sum_{j} b_{i j} X_{j}
$$

For each $i=1, \ldots, n$, there is $\left(p_{i}, q_{i}\right) \neq(0,0)$ such that $\nu=p_{i} \nu_{1}+q_{i} \nu_{2}$ is a non-zero binormal vector and

$$
0=A_{\nu}\left(X_{i}\right)=p_{i} A_{\nu_{1}}\left(X_{i}\right)+q_{i} A_{\nu_{2}}\left(X_{i}\right)=\sum_{j}\left(p_{i} a_{i j}+q_{i} b_{i j}\right) X_{j} .
$$

This implies $p_{i} a_{i j}+q_{i} b_{i j}=0$, for any $i, j$. 
Let $i, j=1, \ldots, n$ and assume that $X_{i}, X_{j}$ are asymptotic directions associated to different binormal vectors, that is, such that

$$
\left|\begin{array}{cc}
p_{i} & p_{j} \\
q_{i} & q_{j}
\end{array}\right| \neq 0 .
$$

Since we have $p_{i} a_{i j}+q_{i} b_{i j}=0$ and $p_{j} a_{i j}+q_{j} b_{i j}=0$, we obtain $a_{i j}=b_{i j}=0$.

Otherwise, if $X_{i}, X_{j}$ are asymptotic directions associated to the same binormal vector, we get

$$
\left|\begin{array}{cc}
p_{i} & p_{j} \\
q_{i} & q_{j}
\end{array}\right|=0
$$

If for instance, $q_{i} \neq 0$, we have $p_{i}=\lambda q_{i}$ and $p_{j}=\lambda q_{j}$, for some $\lambda \in \mathbb{R}$. Since $p_{i} a_{i j}+q_{i} b_{i j}=0$, this gives $b_{i j}=-\lambda a_{i j}$. In general, we will have that either $b_{i j}=\mu a_{i j}$ or $a_{i j}=\lambda b_{i j}$, for some $\lambda, \mu \in \mathbb{R}$.

Therefore, we have showed that the matrices $A=\left(a_{i j}\right)$ and $B=\left(b_{i j}\right)$ have the form:

$$
A=\left(\begin{array}{ccc}
A_{1} & \ldots & 0 \\
\vdots & \ddots & \vdots \\
0 & \ldots & A_{m}
\end{array}\right), \quad B=\left(\begin{array}{ccc}
B_{1} & \ldots & 0 \\
\vdots & \ddots & \vdots \\
0 & \ldots & B_{m}
\end{array}\right)
$$

where $A_{i}, B_{i}$ are submatrices of $A, B$ respectively such that $A_{i}=\lambda_{i} C_{i}$ and $B_{i}=\mu_{i} C_{i}$, where $\lambda_{i}, \mu_{i} \in \mathbb{R}, C_{i}$ is a matrix and $m$ is the number of distinct binormal vectors. Hence,

$$
A B=\left(\begin{array}{ccc}
\lambda_{1} \mu_{1} C_{1}^{2} & \ldots & 0 \\
\vdots & \ddots & \vdots \\
0 & \ldots & \lambda_{m} \mu_{m} C_{m}^{2}
\end{array}\right)=B A
$$

which implies that $R_{D}(p)=0$ by Remark 2.2 .

Remark 3.4. It follows, from the proof of the above theorem, that if $R_{D}(p)=0$ and $\left\{X_{1}, \ldots, X_{n}\right\}$ is an orthonormal frame of $T_{p} M$ made of $\nu$-principal directions, for any $\nu \in N_{p} M$, then $\left\{X_{1}, \ldots, X_{n}\right\}$ are asymptotic directions. The converse is not true in general.

Corollary 3.5. Let $M^{n} \subset \mathbb{R}^{n+2}$ be an immersed submanifold and assume that $R_{D}(p)=0$. Then, there are $n$ binormal directions (counting their multiplicities) at $p$. 


\section{Curvature locus}

We introduce now the concept of curvature locus of an immersed submanifold $M^{n} \subset \mathbb{R}^{n+2}$. When $n=2$, this is nothing but the curvature ellipse at a point $p \in M$ and it contains all the second order information of the immersion.

Definition 4.1. Given $p \in M$, we denote $S_{p}=\left\{X \in T_{p} M,\langle X, X\rangle=1\right\}$. We define the curvature locus, $\Delta_{p}$, as the image set of the map $\eta: S_{p} \rightarrow N_{p} M$ given by $\eta(X)=s(X, X)$.

Let $\left\{\nu_{1}, \nu_{2}\right\}$ and $\left\{X_{1}, \ldots, X_{n}\right\}$ be orthonormal frames of $N_{p} M$ and $T_{p} M$ respectively. Assume that

$$
A_{\nu_{1}}\left(X_{i}\right)=\sum_{j} a_{i j} X_{j}, \quad A_{\nu_{2}}\left(X_{i}\right)=\sum_{j} b_{i j} X_{j}
$$

Given $X \in S_{p}$, we set $X=x_{1} X_{1}+\cdots+x_{n} X_{n}$ with $x_{1}^{2}+\cdots+x_{n}^{2}=1$. Then,

$$
\begin{aligned}
\eta(X) & =s(X, X)=\left\langle A_{\nu_{1}}(X), X\right\rangle \nu_{1}+\left\langle A_{\nu_{2}}(X), X\right\rangle \nu_{2} \\
& =\left(\sum_{i, j} a_{i j} x_{i} x_{j}\right) \nu_{1}+\left(\sum_{i, j} b_{i j} x_{i} x_{j}\right) \nu_{2} .
\end{aligned}
$$

Therefore, $\eta$ is the restriction to the sphere $S_{p}$ of a homogeneous polynomial map of degree 2 (i.e., a quadratic map). If $n=2$, then $\Delta_{p}$ is an ellipse in the normal plane $N_{p} M$. In general, $\Delta_{p}$ is a plane projection of an $(n-1)$ dimensional Veronese manifold.

The following lemma is an easy exercise for quadratic maps in the plane.

Lemma 4.2. Let $h=\left(h_{1}, h_{2}\right): \mathbb{R}^{2} \rightarrow \mathbb{R}^{2}$ be a quadratic map. The ellipse $h\left(S^{1}\right)$ degenerates to a segment $\overline{P Q}$ if and only if $P=h(X), Q=h(Y)$, where $\{X, Y\}$ is an orthonormal frame of $\mathbb{R}^{2}$ which diagonalizes the two quadratic forms $h_{1}, h_{2}$ simultaneously.

Theorem 4.3. Let $M^{n} \subset \mathbb{R}^{n+2}$ be an immersed submanifold and let $p \in M$. Then $R_{D}(p)=0$ if and only if there are $X_{1}, \ldots, X_{n} \in S_{p}$ such that for any $i \neq j, X_{i}, X_{j}$ generate a 2-plane $\pi_{i j} \subset T_{p} M$ and $\eta\left(S_{p} \cap \pi_{i j}\right)$ is the segment $\overline{\eta\left(X_{i}\right) \eta\left(X_{j}\right)}$.

Proof. Assume that $R_{D}(p)=0$ and let $\left\{X_{1}, \ldots, X_{n}\right\}$ be an orthonormal frame of $T_{p} M$ made of $\nu$-principal directions, for any $\nu \in N_{p} M$. Let also $\left\{\nu_{1}, \nu_{2}\right\}$ be an orthonormal frame of $N_{p} M$. Since $\left\{X_{1}, \ldots, X_{n}\right\}$ diagonalize simultaneously the two quadratic forms of $\eta$, the same is true for its restriction to the plane $\pi_{i j}$ and the result follows from the above lemma. 
Conversely, assume that there are $X_{1}, \ldots, X_{n} \in S_{p}$ such that for any $i \neq j, X_{i}, X_{j}$ generate a 2-plane $\pi_{i j} \subset T_{p} M$ and $\eta\left(S_{p} \cap \pi_{i j}\right)=\overline{P_{i} P_{j}}$, where $P_{i}=\eta\left(X_{i}\right)$. The above lemma implies that $X_{1}, \ldots, X_{n}$ is an orthonormal frame of $T_{p} M$ which diagonalizes simultaneously the two quadratic forms of $\eta$. Thus, $R_{D}(p)=0$ by Remark 2.2 .

Corollary 4.4. Let $M^{n} \subset \mathbb{R}^{n+2}$ be an immersed submanifold and $p \in M a$ point such that $R_{D}(p)=0$. Let $\left\{\nu_{1}, \nu_{2}\right\}$ and $\left\{X_{1}, \ldots, X_{n}\right\}$ be orthonormal frames of $N_{p} M$ and $T_{p} M$ respectively, such that

$$
A_{\nu_{1}}\left(X_{i}\right)=\lambda_{i} X_{i}, \quad A_{\nu_{2}}\left(X_{i}\right)=\mu_{i} X_{i}
$$

Then $\Delta_{p}$ is the convex envelope of $P_{1}, \ldots, P_{n}$, where $P_{i}=\eta\left(X_{i}\right)=\lambda_{i} \nu_{1}+\mu_{i} \nu_{2}$.

Proof. The assertion follows easily from the above computations. Just note that given $X \in S_{p}$, we set $X=x_{1} X_{1}+\cdots+x_{n} X_{n}$ with $x_{1}^{2}+\cdots+x_{n}^{2}=1$. Then,

$$
\eta(X)=\left(\sum_{i} \lambda_{i} x_{i}^{2}\right) \nu_{1}+\left(\sum_{i} \mu_{i} x_{i}^{2}\right) \nu_{2}=\sum_{i} x_{i}^{2} P_{i} .
$$

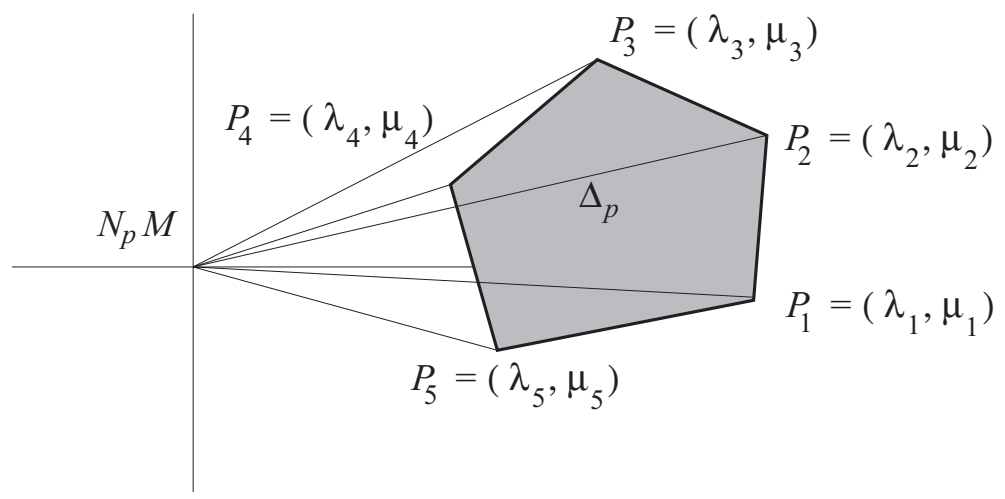

Figure 1:

Example 4.5. We see that the converse of the above corollary is not true in general. Let us consider $g: \mathbb{R}^{5} \rightarrow \mathbb{R}^{7}$ given by

$$
g(x, y, z, t, u)=\left(x, y, z, t, u, 2 x^{2}-2 z^{2}+u^{2},-x^{2}+2 y^{2}-z^{2}+t^{2}+t u\right) .
$$

A simple computation shows that the curvature locus $\Delta_{p}$ at $p=0$ is the triangle with vertices $(2,-1),(0,2)$ and $(-2,-1)$, but $R_{D}(p) \neq 0$. In fact, the restriction to the $(t, u)$-plane gives a non degenerate ellipse contained in the triangle $\Delta_{p}$ (see Figure 2). 


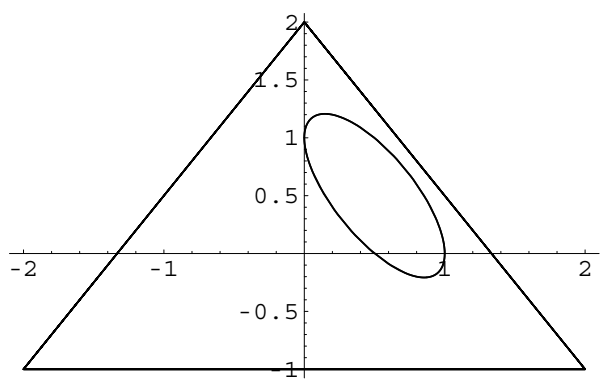

Figure 2:

However, there are some cases where the above construction cannot be done, for instance, when $n=3$, or when $\Delta_{p}$ is a convex polygon with $n$ vertices.

Corollary 4.6. Let $M^{n} \subset \mathbb{R}^{n+2}$ be an immersed submanifold. If $\Delta_{p}$ is a convex $n$-gon, then $R_{D}(p)=0$.

Corollary 4.7. Let $M^{3} \subset \mathbb{R}^{5}$ be an immersed submanifold. Then $R_{D}(p)=0$ if and only if $\Delta_{p}$ is a triangle.

Remark 4.8. Suppose that $R_{D}(p)=0$ and let $\left\{X_{i}\right\}_{i=1}^{n}$ be the common $\nu$-principal directions. Given any normal direction $\nu$ at $p$, the principal curvatures of $A_{\nu}$ are given by $\kappa_{i}^{\nu}=\left\langle\nu, \eta\left(X_{i}\right)\right\rangle, i=1, \ldots, n$. It follows that $\nu$ points in a binormal direction at $p$ if and only if $\nu=\eta\left(X_{i}\right)^{\perp}$. Therefore, we have that the normal vectors $\left\{\eta\left(X_{i}\right)^{\perp}\right\}_{i=1}^{n}$ define the binormal directions at $p$. We observe that we may have $\eta\left(X_{i}\right)= \pm \eta\left(X_{j}\right)$ with $i \neq j$, in such case the binormal $\eta\left(X_{i}\right)^{\perp}$ has multiplicity at least 2 .

On the other hand, it follows that the normal vectors orthogonal to the different segments $\eta\left(X_{i}\right)-\eta\left(X_{j}\right)$ have an associated principal curvature of multiplicity at least 2 .

Proposition 4.9. Let $M^{n} \subset \mathbb{R}^{n+2}$ be an immersed submanifold. Let $p \in M$. Suppose that $R_{D}(p)=0$, then $p$ is either umbilic for at least a normal direction, or there exist at least 3 and at most $\frac{1}{2} n(n-1)$ normal directions defining shape operators with some curvature of multitplicity at least 2 at p.

Proof. Since $R_{D}(p)=0$ the curvature locus $\Delta_{p}$ is either a point, a segment or an $r$-gon, for some $r \geq 3$. If $\Delta_{p}$ is a point, then $\eta\left(X_{i}\right)=\eta\left(X_{j}\right), \forall i, j=$ $1, \ldots, n$, where $\left\{X_{i}\right\}_{i=1}^{n}$ are the common $\nu$-principal directions at $p$. In this case it follows from Remark 4.8 that for any normal direction $\nu \in$ $N_{p} M, \kappa_{i}^{\nu}=\left\langle\nu, \eta\left(X_{i}\right)\right\rangle=\left\langle\nu, \eta\left(X_{j}\right)\right\rangle=\kappa_{j}^{\nu}, \forall i, j=1, \ldots, n$. Therefore $p$ is $\nu$-umbilic for any normal direction $\nu$. In case $\Delta_{p}$ is a segment, the vectors 
$\eta\left(X_{i}\right)-\eta\left(X_{j}\right)$ with $i \neq j$ are pairwise linearly dependent. Then the direction $\left(\eta\left(X_{i}\right)-\eta\left(X_{j}\right)\right)^{\perp}$ defines a normal vector $\nu$, such that $\kappa_{i}^{\nu}=\kappa_{j}^{\nu}, \forall i, j=$ $1, \ldots, n$. So $p$ is $\nu$-umbilic (i.e., semiumbilic). Finally, if $\Delta_{p}$ is an $r$-gon, for some $r \geq 3$, the orthogonal directions to either the sides or the diagonals of $\Delta_{p}$ provide the required normal vectors. Since there are at least 3 and at most $\frac{1}{2} n(n-1)$ of such directions we get the required result.

Definition 4.10. A normal vector $\nu$ is said to be quasiumbilic at $p \in M$ if one of its associated principal curvatures has multiplicity $\geq n-1$ at $p$.

Corollary 4.11. Let $M^{n} \subset \mathbb{R}^{n+2}, n \geq 3$ be an immersed submanifold. suppose that $R_{D}(p)=0$. Then if $p$ is a (non semiumbilic) quasiumbilic point, $\Delta_{p}$ is a triangle.

Corollary 4.12. Let $M^{3} \subset \mathbb{R}^{5}$ be an immersed submanifold. Then $R_{D}(p)=0$ if and only if either $p$ is umbilic for at least a normal direction, or quasiumbilic for 3 different normal directions.

An $n$-dimensional submanifold $M$ of $\mathbb{R}^{n+2}$ is said to be quasiumbilical with respect to a normal field $\nu$ if $A_{\nu}$ has some eigenvalue with multiplicity $\geq n-1$ at every point. The manifold $M$ is said to be totally quasiumbilical provided it admits two mutually orthogonal quasiumbilical normal sections. The notion of totally quasiumbilical will appear later in connection with conformal flatness.

Proposition 4.13. Let $M^{n} \subset \mathbb{R}^{n+2}, n \geq 3$ be a totally quasiumbilical submanifold with $R_{D} \equiv 0$. Then the curvature locus at any non semiumbilic point of $M$ is a right-angled triangle.

Proof. If $R_{D} \equiv 0$ over $M$ we have that $\Delta_{p}$ is a polygon at every point of $M$. Suppose now that $M$ is totally quasiumbilical. Then there exist normal fields $\nu_{1}$ and $\nu_{2}$ which are quasiumbilical all over $M$. But this implies that, for each $p \in M$, there is an $(n-2)$-subspace of $T_{p} M$ made of principal directions for any normal field on $M$. In other words, it is possible to find $n-2$ mutually orthogonal common principal directions $\left\{X_{i}\right\}_{i=1}^{n-2}$ for all the normal fields on $M$ such that $\eta\left(X_{i}\right)=\eta\left(X_{j}\right), \forall i, j=1, \ldots, n-2$. Consequently $\Delta_{p}$ is either a point (when $p$ is umbilic), a segment (when $p$ is semiumbilic), or a triangle. Since the fields $\nu_{1}$ and $\nu_{2}$ must be orthogonal to some side of the triangle and they are mutually orthogonal, we have that the triangle must have a right angle.

Corollary 4.14. Let $M^{3} \subset \mathbb{R}^{5}$ be an immersed submanifold. Suppose that $M$ does not have semiumbilic, nor umbilic points. Then $M$ is totally quasiumbilic and has flat normal bundle if and only if the curvature locus at each point is a right-angled triangle. 


\section{Semiumbilical and strictly locally convex submani- folds}

Given an immersed surface $M^{2} \subset \mathbb{R}^{4}$, we have the following equivalent statements for a point $p \in M$ (see [15]):

1. $p$ is semiumbilic,

2. the curvature ellipse at $p$ degenerates to a segment,

3. $R_{D}(p)=0$.

We will see that the equivalence between (1) and (2) remains true when $n \geq 3$, but they are not equivalent to (3) in general. We will also discuss about the relationship of these notions with the local convexity of the submanifold.

Proposition 5.1. Let $M^{n} \subset \mathbb{R}^{n+2}$ be an immersed submanifold. If $p \in M$ is semiumbilic, then $R_{D}(p)=0$.

Proof. Let $\nu_{1} \in N_{p} M$ be a unit normal vector such that $p$ is $\nu_{1}$-umbilic. This means that $A_{\nu_{1}}=\lambda \mathrm{Id}$, for some $\lambda \in \mathbb{R}$. Now we complete $\nu_{1}$ to an orthonormal frame $\left\{\nu_{1}, \nu_{2}\right\}$ of $N_{p} M$. Hence,

$$
A_{\nu_{1}} \circ A_{\nu_{2}}=\lambda A_{\nu_{2}}=A_{\nu_{2}} \circ A_{\nu_{1}}
$$

and thus $R_{D}(p)=0$ by Remark 2.2 .

Corollary 5.2. Let $M^{n} \subset \mathbb{R}^{n+2}$ be an immersed submanifold and let $p \in M$.

1. $p$ is semiumbilic if and only if $\Delta_{p}$ degenerates to a segment. Moreover, if $L_{p}$ is the linear subspace spanned by $\Delta_{p}$ and $\nu$ is a normal vector such that $p$ is $\nu$-umbilic, then $\nu \perp L_{p}$

2. $p$ is umbilic if and only if $\Delta_{p}$ degenerates to a point.

Proof. By the above proposition and Theorem 4.3, we can assume that $R_{D}(p)=0$. Let $\left\{\nu_{1}, \nu_{2}\right\}$ and $\left\{X_{1}, \ldots, X_{n}\right\}$ be orthonormal frames of $N_{p} M$ and $T_{p} M$ respectively, such that

$$
A_{\nu_{1}}\left(X_{i}\right)=\lambda_{i} X_{i}, \quad A_{\nu_{2}}\left(X_{i}\right)=\mu_{i} X_{i}
$$

Moreover, $\Delta_{p}$ is the convex envelope of $P_{1}, \ldots, P_{n}$ in $N_{p} M$, where $P_{i}=$ $\left(\lambda_{i}, \mu_{i}\right)$. 
By definition, $p$ is semiumbilic if there is a non-zero normal vector $\nu \in$ $N_{p} M$ such that $p$ is $\nu$-umbilic. This is equivalent to the fact that there exist $a, b, \alpha \in \mathbb{R}$ such that $a^{2}+b^{2}>0$ and

$$
a A_{\nu_{1}}+b A_{\nu_{2}}=\alpha \mathrm{Id} .
$$

But this happens if and only if the following matrix has rank $\leq 2$

$$
\left(\begin{array}{ccc}
\lambda_{1} & \cdots & \lambda_{n} \\
\mu_{1} & \cdots & \mu_{n} \\
1 & \cdots & 1
\end{array}\right)
$$

which is equivalent to the collinearity of the points $P_{1}, \ldots, P_{n}$. In fact, if $p$ is $\nu$-umbilic, $P_{1}, \ldots, P_{n}$ are contained in the line $L_{p}$ whose equation is $a x+b y=\alpha$, which is perpendicular to $\nu$.

To see the second part, it follows from the definition that $p$ is umbilic if and only if $\lambda_{1}=\cdots=\lambda_{n}$ and $\mu_{1}=\cdots=\mu_{n}$, which is equivalent to $P_{1}=\cdots=P_{n}$.

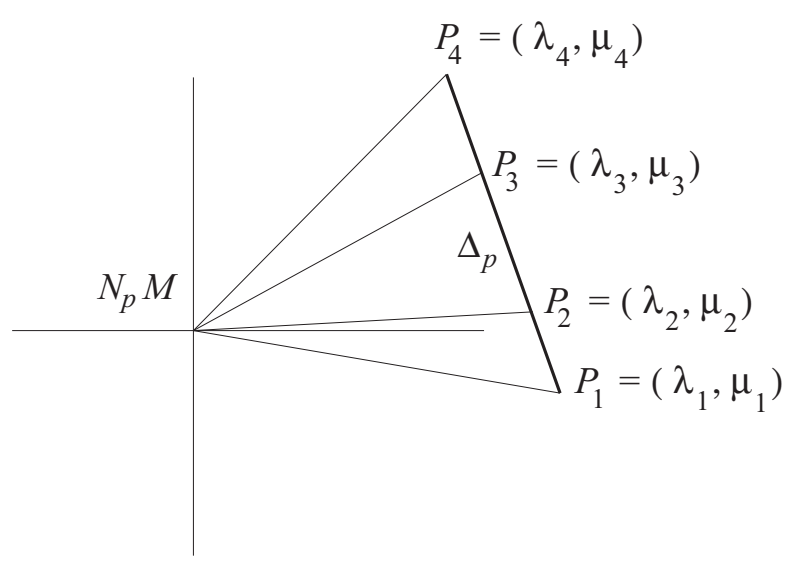

Figure 3:

In the last part of this section, we will see that semiumbilicity is related to the local convexity of $M$ in $\mathbb{R}^{n+2}$.

Definition 5.3. We say that $M$ is locally convex at $p$ if there is a hyperplane $\pi$ of $\mathbb{R}^{n+2}$ such that $p \in \pi$ and $\pi$ supports $M$ in a neighbourhood of $p$. If $\pi$ locally supports $M$ at $p$, it is obvious that $\pi$ is tangent to $M$ at $p$. If it has a non-degenerate contact (i.e., of Morse type), then we say that $M$ is strictly locally convex at $p$. This happens if and only if there is $\nu \in N_{p} M$ such that all the $\nu$-principal curvatures are positive (or all of them are negative). In particular, this gives the following immediate consequence. 
Proposition 5.4. Let $M^{n} \subset \mathbb{R}^{n+2}$ be an immersed submanifold. If $p \in M$ is semiumbilic and has non-zero $\nu$-principal curvature (where $p$ is $\nu$-umbilic), then $M$ is strictly locally convex at $p$.

Proposition 5.5. Let $M^{n} \subset \mathbb{R}^{n+2}$ be an immersed submanifold. If $M$ is strictly locally convex at $p$, then there are $n$ binormal directions at $p$ (counting multiplicity).

Proof. Let $\left\{\nu_{1}, \nu_{2}\right\}$ and $\left\{X_{1}, \ldots, X_{n}\right\}$ be orthonormal frames of $N_{p} M$ and $T_{p} M$ respectively, such that all the $\nu_{1}$-principal curvatures are positive and $\left\{X_{1}, \ldots, X_{n}\right\}$ are $\nu_{1}$-principal directions. This means that

$$
A_{\nu_{1}}\left(X_{i}\right)=\lambda_{i} X_{i}, \quad A_{\nu_{2}}\left(X_{i}\right)=\sum_{j} b_{i j} X_{j},
$$

with $\lambda_{i}>0$ and $b_{i j}=b_{j i}$. Let $\Lambda, C$ be the diagonal matrices with diagonal entries $\lambda_{1}, \ldots, \lambda_{n}$ and $1 / \sqrt{\lambda_{1}}, \ldots, 1 / \sqrt{\lambda_{n}}$ respectively and let us also denote $B=\left[b_{i j}\right]$.

Given a non-zero normal vector $\nu=p \nu_{1}+q \nu_{2} \in N_{p} M$, it is binormal if and only if

$$
\operatorname{det}\left(A_{\nu}\right)=\operatorname{det}\left(p A_{\nu_{1}}+q A_{\nu_{2}}\right)=0 .
$$

This is equivalent in coordinates to

$$
0=\operatorname{det}(p \Lambda+q B) \Longleftrightarrow 0=\operatorname{det}(C(p \Lambda+q B) C)=\operatorname{det}\left(p I_{n}+q \bar{B}\right),
$$

where $I_{n}$ is the identity matrix and $\bar{B}=C B C$.

Note that if $\nu$ is binormal, then $q \neq 0$ (otherwise, $q=0$ would also imply $p=0)$ and $-p / q$ is an eigenvalue of $\bar{B}$. Conversely, if $t$ is an eigenvalue of $\bar{B}$, then $\nu=-t \nu_{1}+\nu_{2}$ is binormal. Moreover, since $\bar{B}$ is symmetric, it has $n$ real eigenvalues (counting multiplicity).

Remark 5.6. We have the following implications:

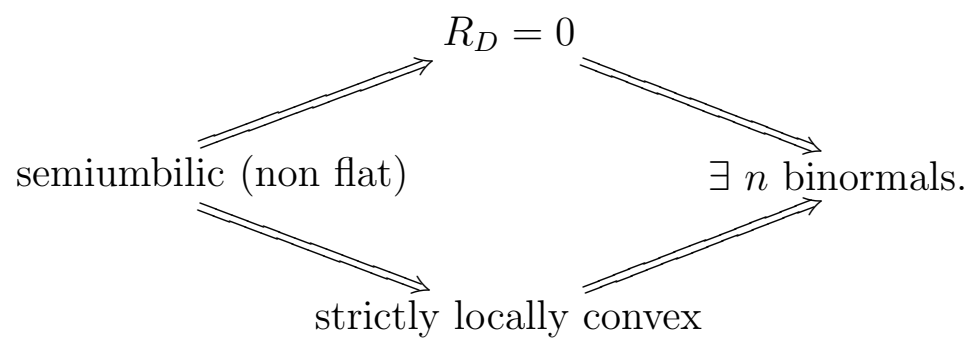

When $n=2$, we have that

$$
\begin{aligned}
\text { semiumbilic } & \Longleftrightarrow R_{D}=0, \\
\text { strictly locally convex } & \Longleftrightarrow \exists 2 \text { binormals. }
\end{aligned}
$$


However, when $n \geq 3$, these equivalences are not true in general. For instance, let $M$ be the 3-manifold embedded in $\mathbb{R}^{5}$, which is parametrized by the map $g: \mathbb{R}^{3} \rightarrow \mathbb{R}^{5}$ defined as

$$
g(x, y, z)=\left(x, y, z, x^{2}-z^{2}, y^{2}-z^{2}\right)
$$

It is not difficult to see that $M$ is not strictly locally convex at $p=0$, although it has vanishing normal curvature at this point.

Another difference with the case $n=2$ occurs with the codimension 2 submanifolds which are a product of two hypersurfaces. If $M=C_{1} \times C_{2} \subset \mathbb{R}^{4}$ is a product of two plane curves, then $M$ is always semiumbilic (and hence has vanishing normal curvature). This is not true in general when $n \geq 3$.

Proposition 5.7. Let $M^{n} \subset \mathbb{R}^{n+1}$ and $P^{m} \subset \mathbb{R}^{m+1}$ be immersed hypersurfaces and consider $M \times P \subset \mathbb{R}^{n+m+2}$. Then $M \times P$ always satisfies $R_{D}=0$. However, $(m, p) \in M \times P$ is semiumbilic if and only if either

1. both $m, p$ are umbilic in $M, P$ respectively, or

2. one of $m, p$ is a flat umbilic in $M, P$ respectively.

Proof. Fix $p=\left(p_{1}, p_{2}\right) \in M \times P$. Let $\nu_{1}$ be a unit normal vector of $M$ in $\mathbb{R}^{n+1}$ at $p_{1}$ and take $\left\{X_{1}, \ldots, X_{n}\right\}$ an orthonormal frame of $T_{p_{1}} M$ made of principal directions with principal curvatures $\lambda_{1}, \ldots, \lambda_{n}$.

Analogously, we consider $\nu_{2}$ a unit normal vector of $P$ in $\mathbb{R}^{m+1}$ at $p_{2}$ and $\left\{Y_{1}, \ldots, Y_{m}\right\}$ an orthonormal frame of $T_{p_{2}} P$ made of principal directions with principal curvatures $\mu_{1}, \ldots, \mu_{m}$.

We identify

$$
N_{p}(M \times P) \equiv N_{p_{1}} M \oplus N_{p_{2}} P \quad \text { and } \quad T_{p}(M \times P) \equiv T_{p_{1}} M \oplus T_{p_{2}} P .
$$

Then $\left\{\nu_{1}, \nu_{2}\right\}$ and $\left\{X_{1}, \ldots, X_{n}, Y_{1}, \ldots, Y_{m}\right\}$ are orthonormal frames of $N_{p}(M \times P)$ and $T_{p}(M \times P)$ respectively, which verify

$$
A_{\nu_{1}}\left(X_{i}\right)=\lambda_{i} X_{i}, \quad A_{\nu_{1}}\left(Y_{j}\right)=0, \quad A_{\nu_{2}}\left(X_{i}\right)=0, \quad A_{\nu_{2}}\left(Y_{j}\right)=\mu_{j} Y_{j} .
$$

This shows that $R_{D}(p)=0$ by Remark 2.2 .

To see the second part, we use Corollary 5.2. In fact, $p$ is semiumbilic if and only if $P_{1}, \ldots, P_{n}, Q_{1}, \ldots, Q_{m}$ are collinear, where $P_{i}=\left(\lambda_{i}, 0\right)$ and $Q_{j}=\left(0, \mu_{j}\right)$. But this can only happen in one the following cases:

1. $P_{1}=\cdots=P_{n}$ and $Q_{1}=\cdots=Q_{m}$,

2. $P_{1}=\cdots=P_{n}=0$, or

3. $Q_{1}=\cdots=Q_{m}=0$. 


\section{Parallel normal vector fields and parallel submani- folds}

In this section we consider an immersed submanifold $M$ which locally admits a non-degenerate parallel normal vector field $\nu$. This kind of submanifold have been introduced in [11] where it is shown that the contact of $M$ with hyperplanes is the same as the contact of the parallel hyperspherical submanifold $M^{\nu}$ with the translated hyperplane. In the codimension 2 case, the existence of a parallel normal vector field is equivalent to the flatness of the normal bundle and the non-degeneracy of such a vector field is related to the non flatness in some sense of the tangent bundle.

Definition 6.1. We say that a normal vector field $\nu$ is parallel if $D_{X} \nu=0$ for any $X \in T_{p} M$ and any $p \in M$.

The proof of the following lemma can be found in [12] in a more general version for a codimension $k$ submanifold $M^{n} \subset \mathbb{R}^{n+k}$.

Lemma 6.2. Let $M^{n} \subset \mathbb{R}^{n+2}$ be an immersed submanifold. The following conditions are equivalent:

1. $R_{D}=0$ in $M$.

2. There is a locally defined non-zero parallel normal vector field $\nu$.

3. There are locally defined parallel normal vector fields $\nu_{1}, \nu_{2}$ which provide an orthonormal frame of $N_{p} M$ at any point $p \in M$.

We note that if a normal vector field is parallel, then it has constant length. Moreover, if $M^{n} \subset \mathbb{R}^{n+2}$ and $\nu$ is parallel then $\nu^{\perp}$ is also parallel.

Definition 6.3. We say that a normal vector $\nu \in N_{p} M$ is non-degenerate if $\operatorname{det} A_{\nu} \neq 0$, that is, if $\nu$ is not binormal. We say that a normal vector field is non-degenerate if it is non-degenerate at any point $p \in M$.

Let $M^{n} \subset \mathbb{R}^{n+2}$ be an immersed submanifold and assume that $R_{D}=0$ at $p \in M$. We take $\left\{\nu_{1}, \nu_{2}\right\}$ and $\left\{X_{1}, \ldots, X_{n}\right\}$ orthonormal frames of $N_{p} M$ and $T_{p} M$ respectively such that

$$
A_{\nu_{1}}\left(X_{i}\right)=\lambda_{i} X_{i}, \quad A_{\nu_{2}}\left(X_{i}\right)=\mu_{i} X_{i} .
$$

We denote by $P_{i}$ the vertices of the curvature locus, that is, $P_{i}=\eta\left(X_{i}\right)=$ $\lambda_{i} \nu_{1}+\mu_{i} \nu_{2}$, for each $i=1, \ldots, n$. For any normal vector $\nu=a \nu_{1}+b \nu_{2} \in$ $N_{p} M$, we have that

$$
\operatorname{det} A_{\nu}=\left(a \lambda_{1}+b \mu_{1}\right) \ldots\left(a \lambda_{n}+b \mu_{n}\right) .
$$

Hence, there is a non-degenerate normal vector if and only if $P_{i} \neq 0$, for all $i=1, \ldots, n$. This fact together with lemma 6.2 gives the following result. 
Corollary 6.4. There is a non-degenerate parallel normal vector field defined locally in a neighbourhood of each point $p \in M$ if and only if $R_{D}=0$ and $P_{i} \neq 0$, for all $i=1, \ldots, n$.

In general it is imposible to detect intrinsically the vanishing of a vertex $P_{i}$ as the following example shows.

Example 6.5. Consider the product $M=\Gamma \times Q$ with $\Gamma \subset \mathbb{R}^{2}$ a plane curve with non-vanishing geodesic curvature $\kappa$ and $Q \subset \mathbb{R}^{n}$ a hypersurface with non-vanishing principal curvatures $k_{1}, \ldots, k_{n-1}$. If $L \subset \mathbb{R}^{2}$ is a straight line and $f: \Gamma \rightarrow L$ is a map which preserves arclength, then $F=f \times \operatorname{Id}: M \rightarrow$ $M^{\prime}$ is an isometry from $M$ to the cylinder $M^{\prime}=L \times Q$.

The normal bundle of $M$ has an orthonormal frame $\left\{\nu_{1}, \nu_{2}\right\}$ where $\nu_{1}$ is the normal of $\Gamma$ in $\mathbb{R}^{2}$ and $\nu_{2}$ is the normal of $Q$ in $\mathbb{R}^{n}$. The coordinates of the vertices of the curvature locus of $M$ are $(\kappa, 0)$ and $\left(0, k_{1}\right), \ldots,\left(0, k_{n-1}\right)$. Hence $M$ has a non-degenerate parallel normal vector field defined locally (take for instance $\nu=\nu_{1}+\nu_{2}$ ).

For the cylinder $M^{\prime}$ we have a similar normal frame $\left\{\nu_{1}, \nu_{2}\right\}$ but then the vertices are $(0,0)$ and $\left(0, k_{1}\right), \ldots,\left(0, k_{n-1}\right)$. At any point of $M^{\prime}$ (isometric with $M)$ one has only degenerate normals.

We consider now the curvature operator of a Riemannian manifold $M$, which is the endomorphism $\Phi: T_{p} M \wedge T_{p} M \rightarrow T_{p} M \wedge T_{p} M$. We recall that given $X, Y \in T_{p} M$, then $\Phi(X \wedge Y)$ is the unique bivector such that

$$
\langle\Phi(X \wedge Y), Z \wedge W\rangle=\langle R(X, Y) Z, W\rangle,
$$

for all $Z, W \in T_{p} M$ and where $R: T_{p} M \times T_{p} M \times T_{p} M \rightarrow T_{p} M$ is the curvature tensor of $M$. We shall call the determinant $K=\operatorname{det} \Phi$ the Gaussian curvature of $M$ at $p$.

In the case that $M^{n} \subset \mathbb{R}^{n+2}$ is an immersed submanifold with $R_{D}=0$ at $p \in M$, we take again $\left\{\nu_{1}, \nu_{2}\right\}$ and $\left\{X_{1}, \ldots, X_{n}\right\}$ orthonormal frames of $N_{p} M$ and $T_{p} M$ respectively such that

$$
A_{\nu_{1}}\left(X_{i}\right)=\lambda_{i} X_{i}, \quad A_{\nu_{2}}\left(X_{i}\right)=\mu_{i} X_{i}
$$

and we denote by $P_{i}=\eta\left(X_{i}\right) i=1, \ldots, n$ the vertices of the curvature locus.

Lemma 6.6. With the above notation, the eigenvectors of the curvature operator $\Phi$ are the simple elements $X_{i} \wedge X_{j}, i<j$, with corresponding eigenvalues $-\left\langle P_{i}, P_{j}\right\rangle$. In particular,

$$
K=\operatorname{det} \Phi=(-1)^{\frac{n(n-1)}{2}} \prod_{i<j}\left\langle P_{i}, P_{j}\right\rangle .
$$


Proof. This is an easy consequence of Gauss Equation:

$$
\begin{aligned}
\left\langle R\left(X_{i}, X_{j}\right) Z, W\right\rangle= & \left\langle\lambda_{i} X_{i}, W\right\rangle\left\langle\lambda_{j} X_{j}, Z\right\rangle-\left\langle\lambda_{i} X_{i}, Z\right\rangle\left\langle\lambda_{j} X_{j}, W\right\rangle \\
& +\left\langle\mu_{i} X_{i}, W\right\rangle\left\langle\mu_{j} X_{j}, Z\right\rangle-\left\langle\mu_{i} X_{i}, Z\right\rangle\left\langle\mu_{j} X_{j}, W\right\rangle \\
= & -\left(\lambda_{i} \lambda_{j}+\mu_{i} \mu_{j}\right)\left\langle X_{i} \wedge X_{j}, Z \wedge W\right\rangle,
\end{aligned}
$$

and so $\Phi\left(X_{i} \wedge X_{j}\right)=-\left\langle P_{i}, P_{j}\right\rangle X_{i} \wedge X_{j}$.

As a consequence, if $K \neq 0$ then $\left\langle P_{i}, P_{j}\right\rangle \neq 0$ for all $i<j$ and hence, $P_{i} \neq 0$ for all $i$. This gives the following corollary.

Corollary 6.7. Let $M^{n} \subset \mathbb{R}^{n+2}$ be an immersed submanifold. If $R_{D}=0$ and $K \neq 0$ in $M$, then there is a non-degenerate parallel normal vector field defined locally in a neighbourhood of each point $p \in M$.

Remark 6.8. Let $M^{n} \subset \mathbb{R}^{n+1}$ be a hypersurface with principal curvatures $\lambda_{1}, \ldots, \lambda_{n}$. We can consider $M^{n} \subset \mathbb{R}^{n+1} \subset \mathbb{R}^{n+2}$ in such a way that it is an immersed submanifold with vanishing normal curvature. We put $\mu_{i}=0$ and the above lemma gives that

$$
K=(-1)^{\frac{n(n-1)}{2}} \prod_{i<j}\left(\lambda_{i} \lambda_{j}\right)=(-1)^{\frac{n(n-1)}{2}}\left(\lambda_{1} \ldots \lambda_{n}\right)^{2} .
$$

We recall that the product $\lambda_{1} \ldots \lambda_{n}$ is the classical Gaussian curvature of the hypersurface.

The above corollary can be improved. In fact, we find conditions much weaker than invertibility of $\Phi$ which also give the non-vanishing of the vertices $P_{i}$.

Lemma 6.9. With the notation of lemma 6.6, the kernel of the curvature operator contains a product $X \wedge T_{p} M$ if and only if at least one of the $P_{i}$ is orthogonal to all other vertices $P_{j}$.

Proof. If $P_{i_{0}}$ is orthogonal to $P_{j}, j \neq i_{0}$, then $X_{i_{0}} \wedge T_{p} M$ is an $(n-1)$ dimensional subspace of $\operatorname{ker} \Phi$. Assume conversely that $\operatorname{ker} \Phi$ contains a product $X \wedge T_{p} M$, and write $X=c_{i_{1}} X_{i_{1}}+\cdots+c_{i_{s}} X_{i_{s}}$ for some indices $i_{1}<\cdots<i_{s}$ and non-zero coefficients $c_{i_{1}}, \ldots, c_{i_{s}}$. Then, for every $j$ we have:

$$
0=\Phi\left(X \wedge X_{j}\right)=-\sum_{i_{h} \neq j} c_{i_{h}}\left\langle P_{i_{h}}, P_{j}\right\rangle X_{i_{h}} \wedge X_{j}
$$

Letting $j$ run from 1 to $n$ and using the linear independence of the products $X_{i_{h}} \wedge X_{j}$, we conclude that each $P_{i_{h}}$ is orthogonal to all other vertices $P_{j}$.

The condition $X \wedge T_{p} M \subset \operatorname{ker} \Phi$ implies that all 2-planes containing $X$ have zero sectional curvature. It also implies that $\operatorname{Ric}(X, Y)=0$, for all $Y \in T_{p} M$, where Ric is the Ricci bilinear form. 
Corollary 6.10. Let $M^{n} \subset \mathbb{R}^{n+2}$ be an immersed submanifold with $R_{D}=0$. If the Ricci bilinear form of $M$ at $p$ is non-degenerate, or if every direction in $T_{p} M$ is contained in some 2-plane with non-zero sectional curvature, then there is a non-degenerate parallel normal vector field defined locally in a neighbourhood of $p$.

We have also the following condition, which is weaker than $K \neq 0$ for $n \geq 3$.

Corollary 6.11. Let $M^{n} \subset \mathbb{R}^{n+2}$ be an immersed submanifold with $R_{D}=0$. If $\operatorname{dim} \operatorname{ker} \Phi \leq n-2$ at $p \in M$, then there is a non-degenerate parallel normal vector field defined locally in a neighbourhood of $p$.

Definition 6.12. Given a unit normal vector field $\nu \in \mathfrak{X}(M)^{\perp}$, we can consider the Gauss map $G_{\nu}: M \rightarrow S^{n+1}$. For each point $p \in M, G(p)$ is the unit normal vector $\nu_{p}$ translated to the origin of $\mathbb{R}^{n+2}$.

Lemma 6.13. Let $M^{n} \subset \mathbb{R}^{n+2}$ be an immersed submanifold and let $\nu \in$ $\mathfrak{X}(M)^{\perp}$ be a unit normal vector field. We denote by $D_{p} G_{\nu}: T_{p} M \rightarrow T_{\nu_{p}} S^{n+1}$ the differential of $G_{\nu}$ at $p \in M$.

1. $\nu$ is parallel if and only if $D_{p} G_{\nu}\left(T_{p} M\right) \subset T_{p} M$, for each $p \in M$.

2. $\nu$ is parallel and non-degenerate if and only if $D_{p} G_{\nu}\left(T_{p} M\right)=T_{p} M$, for each $p \in M$.

Proof. Given $X \in T_{p} M$, we use Weingarten Equation:

$$
D_{p} G_{\nu}(X)=\nabla_{X}^{\prime} \nu=-A_{\nu}(X)+D_{X} \nu
$$

Hence, $D_{p} G_{\nu}(X) \in T_{p} M$ if and only if $D_{X} \nu=0$. This proves the first part.

For the second part, if $\nu$ is parallel, then $D_{p} G_{\nu}(X)=-A_{\nu}(X)$, for any $X \in T_{p} M$. Therefore, $D_{p} G_{\nu}\left(T_{p} M\right)=T_{p} M$ if and only if $A_{\nu}\left(T_{p} M\right)=T_{p} M$ if and only if $\operatorname{det} A_{\nu} \neq 0$.

Definition 6.14. Let $\nu \in \mathfrak{X}(M)^{\perp}$ be a non-degenerate parallel unit normal vector field. By the above lemma, the Gauss map $G_{\nu}: M \rightarrow S^{n+1}$ is an immersion. We will denote its image by $M^{\nu}=G_{\nu}(M)$ and call it parallel submanifold to $M$ with respect to $\nu$. It follows that $M^{\nu}$ is an immersed submanifold in the sphere $S^{n+1}$ which verifies:

$$
T_{\nu_{p}} M^{\nu}=T_{p} M, \quad N_{\nu_{p}} M^{\nu}=N_{p} M,
$$

for any $p \in M$. 
Lemma 6.15. Let $M^{n} \subset \mathbb{R}^{n+2}$ be an immersed submanifold and let $\nu \in$ $\mathfrak{X}(M)^{\perp}$ be a non-degenerate parallel unit normal vector field. We denote by $A_{\xi}^{\nu}$ the shape operator of the parallel submanifold $M^{\nu}$. Then,

$$
A_{\xi}^{\nu}=-A_{\xi} \circ A_{\nu_{p}}^{-1}
$$

for any $\xi \in N_{p} M$ and any $p \in M$.

Proof. We denote by $s^{\nu}(X, Y)$ the second fundamental form of $M^{\nu}$. We compare it with the second fundamental form $s(X, Y)$ of $M$. Let $X, Y$ be tangent vectors in $T_{p} M=T_{p} M^{\nu}$. Recall that

$$
s^{\nu}(X, Y)=\left(\nabla_{X}^{\prime} \tilde{Y}\right)^{\perp}, \quad s(X, Y)=\left(\nabla_{X}^{\prime} \bar{Y}\right)^{\perp},
$$

where $\tilde{Y}, \bar{Y}$ are local extensions of $Y$ in $M^{\nu}, M$ respectively in a neighbourhood of $\nu_{p}, p$ respectively.

We can write

$$
\tilde{Y}=\sum_{i=1}^{n+2} f_{i} \frac{\partial}{\partial x_{i}},
$$

where $f_{i}$ are functions locally defined in a neighbourhood of $\nu_{p}$ in $M^{\nu}$ and $x_{1}, \ldots, x_{n+2}$ denote the coordinates of $\mathbb{R}^{n+2}$. Then,

$$
\bar{Y}=\sum_{i=1}^{n+2}\left(f_{i} \circ G_{\nu}\right) \frac{\partial}{\partial x_{i}},
$$

defines a local extension of $Y$ in $M$. By using these extensions, we get

$$
\begin{aligned}
s(X, Y) & =\left(\nabla_{X}^{\prime} \bar{Y}\right)^{\perp} \\
& =\left(\sum_{i=1}^{n+2} X\left(f_{i} \circ G_{\nu}\right) \frac{\partial}{\partial x_{i}}\right)^{\perp} \\
& =\left(\sum_{i=1}^{n+2} D_{p} G_{\nu}(X)\left(f_{i}\right) \frac{\partial}{\partial x_{i}}\right)^{\perp} \\
& =\left(\nabla_{D_{p} G_{\nu}(X)}^{\prime}\right)^{\perp} \\
& =s^{\nu}\left(D_{p} G_{\nu}(X), Y\right) \\
& =-s^{\nu}\left(A_{\nu}(X), Y\right) .
\end{aligned}
$$

This shows that $s(X, Y)=-s^{\nu}\left(A_{\nu}(X), Y\right)$ or equivalently, that

$$
s^{\nu}(X, Y)=-s^{\nu}\left(A_{\nu}^{-1}(X), Y\right),
$$

for any $X, Y \in T_{p} M$. 
Finally, given $\xi \in N_{p} M$,

$$
\left\langle A_{\xi}^{\nu}(X), Y\right\rangle=\left\langle s^{\nu}(X, Y), \xi\right\rangle=-\left\langle s^{\nu}\left(A_{\nu}^{-1}(X), Y\right), \xi\right\rangle=-\left\langle A_{\xi}\left(A_{\nu}^{-1}(X)\right), Y\right\rangle,
$$

hence $A_{\xi}^{\nu}=-A_{\xi} \circ A_{\nu_{p}}^{-1}$.

We finish this section with the following theorem which has been proved by the first author in [11] in the case $n=2$. An interesting consequence of it was the extension of the Carathéodory and Loewner conjectures for totally semiumbilic surfaces in $\mathbb{R}^{4}$ with non-zero Gaussian curvature.

Theorem 6.16. Let $M^{n} \subset \mathbb{R}^{n+2}$ be an immersed submanifold and let $\nu \in \mathfrak{X}(M)^{\perp}$ be a non-degenerate parallel unit normal vector field. Then the binormal and asymptotic directions of $M$ at $p$ coincide with the binormal and asymptotic directions of $M^{\nu}$ at $\nu_{p}$.

Proof. Let $p \in M$ and let $\left\{X_{1}, \ldots, X_{n}\right\}$ be an orthonormal frame of $T_{p} M$ made of $\xi$-principal directions, for any $\xi \in N_{p} M$. We also take $\left\{\nu_{1}, \nu_{2}\right\}$ an orthonormal frame of $N_{p} M$ such that $\nu_{1}=\nu_{p}$. Then,

$$
A_{\nu_{1}}\left(X_{i}\right)=\lambda_{i} X_{i}, \quad A_{\nu_{2}}\left(X_{i}\right)=\mu_{i} X_{i}
$$

with $\lambda_{i} \neq 0$. We know that $\left\{X_{1}, \ldots, X_{n}\right\}$ are the asymptotic directions of $M$ and the corresponding binormal vectors are given by

$$
\xi_{i}=\frac{\mu_{i}}{\lambda_{i}} \nu_{1}-\nu_{2}, \quad i=1, \ldots, n
$$

In the parallel submanifold $M^{\nu}$ at $\nu_{p}$ we consider the same frames $\left\{X_{1}, \ldots, X_{n}\right\}$ and $\left\{\nu_{1}, \nu_{2}\right\}$. By the above lemma, we obtain

$$
\begin{aligned}
& A_{\nu_{1}}^{\nu}\left(X_{i}\right)=-A_{\nu_{1}} \circ A_{\nu_{1}}^{-1}\left(X_{i}\right)=-X_{i}, \\
& A_{\nu_{2}}^{\nu}\left(X_{i}\right)=-A_{\nu_{2}} \circ A_{\nu_{1}}^{-1}\left(X_{i}\right)=-\frac{\mu_{i}}{\lambda_{i}} X_{i} .
\end{aligned}
$$

Therefore, we get that the asymptotic directions of $M^{\nu}$ are again $\left\{X_{1}, \ldots, X_{n}\right\}$ with binormal vectors $\left\{\xi_{1}, \ldots, \xi_{n}\right\}$.

We denote by $\pi: S^{n+1} \backslash\{q\} \rightarrow \mathbb{R}^{n+1}$ the stereographical projection with respect to some point $q \in S^{n+1}$. This is a diffeomorphism which is also a conformal map. Assume that $M^{n} \subset \mathbb{R}^{n+2}$ is an immersed submanifold and let $\nu \in \mathfrak{X}(M)^{\perp}$ be a non-degenerate parallel unit normal vector field. We can consider the immersion $\pi \circ G_{\nu}: M \rightarrow \mathbb{R}^{n+1}$ and we denote its image by $\tilde{M}^{\nu}$, which is an immersed hypersurface in $\mathbb{R}^{n+1}$. Moreover, given $p \in M$ and $X \in T_{p} M$ we also denote $\tilde{p}=\pi\left(\nu_{p}\right) \in \tilde{M}^{\nu}$ and $\tilde{X}=D_{p}\left(\pi \circ G_{\nu}\right)(X) \in T_{\tilde{p}} \tilde{M}^{\nu}$. 
Corollary 6.17. Let $M^{n} \subset \mathbb{R}^{n+2}$ be an immersed submanifold and let $\nu \in$ $\mathfrak{X}(M)^{\perp}$ be a non-degenerate parallel unit normal vector field. Then $X \in$ $T_{p} M$ is an asymptotic direction of $M$ at $p$ if and only $\tilde{X}$ is a principal direction of $\tilde{M}^{\nu}$ at $\tilde{p}$.

Proof. This is a consequence of Theorem 6.16 and the fact that the stereographical projection is a conformal map and hence it transforms asymptotic directions of a submanifold $N \subset S^{n+1}$ into principal directions of its image $\pi(N) \subset \mathbb{R}^{n+1}$ (see [8] for details).

\section{Semiumbilicity, hypersphericity and conformal flat- ness}

Given an $n$-manifold $M$ lying in a hypersphere of $\mathbb{R}^{n+2}$, the radius vector is an umbilic normal field over $M$, so $M$ is semiumbilical and hence has vanishing normal curvature. On the other hand, submanifolds with vanishing normal curvature, or even semiumbilical submanifolds, do not need to lie in a hypersphere in general. The connection between semiumbilicity and hypersphericity for surfaces in 4-space was investigated in [15], where necessary and sufficient conditions for semiumbilical surfaces to be hyperspherical in terms of the binormals and the curvature ellipses were obtained. We shall use here some results due to Chen and Yano ([1], [2], [3]) and Chen and Vestraelen $([4])$, together with our previous results, in order to characterize the hypersphericity and the conformal flatness in terms of the curvature locus at each point.

We first observe that Chen and Yano [1] proved that an $n$-manifold $M$ immersed in $\mathbb{R}^{n+2}$ is hyperspherical if and only if $M$ is $\nu$-umbilic for some parallel normal field $\nu$. Therefore we can assert:

Corollary 7.1. Let $M$ be an $n$-manifold $M$ immersed in $\mathbb{R}^{n+2}, n \geq 2$ with isolated umbilic points. Then $M$ is hyperspherical if and only if the curvature locus at every point of $M$ is a segment defining a parallel field off the umbilic set of $M$.

Proof. We have seen that the curvature locus is a segment at a point $p \in M$ if and only if $p$ is $\nu$-umbilic for some field $\nu$. Moreover, $\nu(p)$ is orthogonal to the direction defined by the segment in the normal plane of $M$ at $p$. The assertion follows now easily over the open and dense submanifold determined by the complement of the umbilic points from Chen and Yano's result and is extended to the whole $M$ by continuity. 
Remark 7.2. In the case of a submanifold $M$ with vanishing normal curvature, if $\left\{X_{i}\right\}_{i=1}^{n}$ represents the orthonormal basis of common principal directions on $M$, then the condition above is equivalent to asking that the normal fields $\eta\left(X_{i}\right)-\eta\left(X_{j}\right)$ be pairwise linearly dependent, for all $i \neq j$, and that the unit normal vector field $\nu$ orthogonal to $\eta\left(X_{i}\right)-\eta\left(X_{j}\right)$ is parallel. Moreover, all points $\left\{\eta\left(X_{i}\right)\right\}_{i=1}^{n}$ lie in a parallel field of affine lines defined by $\langle\nu, \cdot\rangle=$ constant in the normal bundle.

We recall now some facts concerning the relations between quasiumbilicity and conformal flatness. Chen and Yano [2] proved that any totally quasiumbilical $n$-manifold with $n \geq 4$ is conformally flat. On the other hand, Chen and Verstraelen [4] proved that any $n$-manifold $M$ which is conformally flat and has flat normal connection in $\mathbb{R}^{n+p}$ is totally quasiumbilical, provided $n>4$ and $p<n-2$. Therefore we can state:

If $M$ is an $n$-manifold with vanishing normal curvature in $\mathbb{R}^{n+2}, n>4$, then

$M$ is conformally flat $\Longleftrightarrow M$ is totally quasiumbilical.

Moreover, Chen and Yano [3] proved that if $M$ is $v$-umbilic for some non parallel normal field $v$, then $M$ is totally quasiumbilical. Here, saying that $v$ is non parallel means that for each $p \in M$, there is a tangent vector $X \in T_{p} M$ such that $D_{X} v \neq 0$. It then follows that totally semiumbilic $n$-manifolds immersed in $\mathbb{R}^{n+2}, n>4$, whose curvature segments determine non parallel normal fields, are conformally flat. Consequently, we can state:

Corollary 7.3. Let $M$ be an n-manifold immersed in $\mathbb{R}^{n+2}, n>4$ and suppose that the curvature locus degenerates to a segment at every point of $M$. Consider a unit normal field $v$ determined by the curvature segment (locally defined) at each point. If $v$ is non parallel, then $M$ is conformally flat.

Summarizing, we have:

Corollary 7.4. Let $M$ be a semiumbilical $n$-manifold immersed in $\mathbb{R}^{n+2}$, $n>4$ with a finite subset of umbilic points $\left\{p_{1}, \ldots, p_{k}\right\}$. Let $\left\{X_{i}\right\}_{i=1}^{n}$ be the orthonormal basis of common principal directions on $M$. Denote $\nu_{i j}=\eta\left(X_{i}\right)-\eta\left(X_{j}\right), i \neq j$. The vectors $\left\{\nu_{i j}\right\}_{i, j=1}^{n}, i \neq j$ do not vanish simultaneously at each $p \notin\left\{p_{1}, \ldots, p_{k}\right\}$ and define a unit normal direction $\nu$ over $M-\left\{p_{1}, \ldots, p_{k}\right\}$. Then we have:

a) If $\nu$ is a parallel field, then $M$ is hyperspherical.

b) If $\nu$ is a non parallel field, then $M$ is conformally flat. 
Acknowledgements: The authors would like to thank the referee for valuable comments and suggestions.

\section{References}

[1] Chen, B.-Y. And Yano, K.: Integral formulas for submanifolds and their applications. J. Differential Geometry 5 (1971), 467-477.

[2] Chen, B.-Y. And Yano, K.: Sous-variété localement conformes à un espace euclidien. C.R. Acad. Sci. Paris Sér. A-B 275 (1972) A123-A125.

[3] Chen, B.-Y. And Yano, K.: Umbilical submanifolds with respect to a nonparallel normal direction. J. Differential Geometry 8 (1973), 589-597.

[4] Chen, B.-Y. and Verstraelen, L.: A characterization of totally quasiumbilical submanifolds and its applications. Bol. Un. Mat. Ital. A (5) $\mathbf{1 4}$ (1977), 49-57.

[5] Garcia, R.A., Mochida, D. K. H., Romero Fuster, M. C. And RUAS, M. A.S.: Inflection points and topology of surfaces in 4-space. Trans. Amer. Math. Soc. 352 (2000), no. 7, 3029-3043.

[6] Little, J.: On singularities of submanifolds of higher dimensional Euclidean space. Ann. Mat. Pura Appl. (4) 83 (1969), 261-335.

[7] Mochida, D. K. H., Romero Fuster, M. C. and Ruas, M. A. S.: The geometry of surfaces in 4-space from a contact viewpoint. Geom. Dedicata 54 (1995), 323-332.

[8] Mochida, D. K.H., Romero Fuster, M. C. and Ruas, M. A. S.: Osculating hyperplanes and asymptotic directions of codimension two submanifolds of Euclidean spaces. Geom. Dedicata 77 (1999), 305-315.

[9] Montaldi, J. A.: Contact with application to submanifolds. PhD Thesis, University of Liverpool, 1983.

[10] Moraes, S. and Romero Fuster, M. C.: Semiumbilics and 2-regular immersions of surfaces in Euclidean spaces. Rocky Mountain J. Math. 35 (2005), no. 4, 1327-1345.

[11] NuÑo-Ballesteros, J. J.: Submanifolds with a non-degenerate parallel normal vector field in Euclidean spaces. In Singularity theory and its applications, 311-332. Adv. Stud. Pure Math. 43. Math. Soc. Japan, Tokyo, 2006.

[12] Palais, R. S. And Terng, C.-L.: Critical point theory and submanifolds geometry. Lecture Notes in Mathematics 1353. Springer-Verlag, Berlin, 1988.

[13] Porteous, I. R.: The normal singularities of a submanifold. J. Differential Geometry 5 (1971), 543-564.

[14] Romero Fuster, M. C.: Stereographic projections and geometric singularities. Workshop on Real and Complex Singularities (São Carlos, 1996). Mat. Contemp. 12 (1997), 167-182. 
[15] Romero Fuster, M. C. And SÁnchez-Bringas, F.: Umbilicity of surfaces with orthogonal asymptotic lines in $\mathbb{R}^{4}$. Differential Geom. Appl. 16 (2002), 213-224.

[16] Terng, C. L.: Submanifolds with flat normal bundle. Math. Ann. 277 (1987), no. 1, 95-111.

[17] Wong, Y. C.: Contributions to the theory of surfaces in a 4-space of constant curvature. Trans. Amer. Math. Soc. 59 (1946), 467-507.

Recibido: 1 de octubre de 2008

Revisado: 7 de abril de 2010

\author{
J. J. Nuño-Ballesteros \\ Departament de Geometria i Topologia \\ Universitat de València \\ 46100 Burjassot (València), Spain \\ juan.nuno@uv.es \\ M. C. Romero-Fuster \\ Departament de Geometria i Topologia \\ Universitat de València \\ 46100 Burjassot (València), Spain \\ carmen.romero@uv.es
}

Work of both authors partially supported by DGCYT and FEDER grant no. MTM200908933 . 